\title{
Image Search: Efficient Image Retrieval Using Color and Texture Features
}

\author{
Mr. Shaikh J.S. ${ }^{1}$, Mr. Khatod S.J. ${ }^{2}$, Miss. Korde S.C. ${ }^{3}$, Miss. Kurhe S.D. ${ }^{4}$ \\ BE Scholar, Department of Computer Engineering, S.R.E.S.'s College of Engineering, Kopargaon, India 1,2,3,4
}

\begin{abstract}
Web-based image search engines mostly rely on surrounding textual features. It becomes very difficult for them to interpret users' search intention only by query keywords and this leads to ambiguous and noisy search results which do not satisfy users perspective. In order to solve this ambiguity in text based image retrieval we used visual information of query image. In this project, we are implementing a novel Internet image search approach where user is asked to click on one query image with less effort and visually relevant images from a huge database are retrieved. Our main perspective is to capture the users' search intention from this one-click query image in following steps. In this system, the user first submits query keyword. A pool of images is retrieved by text-based search. Then the user is asked to select a query image from the image pool. Images in the pool are re-ranked based on their color and texture similarities to the query image. These similarities are computed using Euclidean distance method. A query-specific color similarity metric and a query specific textual similarity metric are learned from the selected examples and used to rank images. These similarity metrics reflect users' intention at a finer level since every query image has different metrics.
\end{abstract}

Keywords: Content based image retrieval (CBIR), Color Coherence Vector (CCV), Texture Element Feature Characterization (TEFC), Pixels, Image, Cluster.

\section{INTRODUCTION}

"Image Search" means that the search will perform using Greg Pass and Ramin Zabih in their paper describe a standard Content Based image Retrieval (CBIR) feature extraction algorithms on the query image to retrieve more relevant images and satisfy users search intention. CBIR is the application of computer vision techniques to the image retrieval problem, that is, the problem of searching for digital images in large databases.

Feature extraction algorithms include color feature extraction and texture feature extraction. Feature extraction is desirable because most web based image search engines rely purely on metadata and this produces a lot of garbage in the results.

Also having humans manually enter keywords for images in a large database can be inefficient, expensive and may not capture exact users search intention. Thus a system that can filter images based on their content would provide better indexing and return more accurate results.

To extract features, CCV (Color Coherence Vector) is used for color feature extraction and TEFC (Texture Element Feature Extraction) is used for texture feature extraction. These CCV and TEFC features of query image are compared with that of database images. These methods' can increase the accuracy and helps the user to obtain the results quickly.

\section{RELATED WORK}

Xiaoou Tang et al in his paper [1] has proposed method for capturing user intention for ine-click internet image search. They proposed intention specific weight schema to combine visual features and to compute visual similarity adaptive to query images. Without additional human color-based method [2] for comparing images which is similar to color histograms, but which also takes spatial information into account. They begin with a review of color histograms. They then describe color coherence vectors (CCV's) and how to compare them.

K. Jalaja and Chakravarthy Bhagvati in their paper propose two characterizations of local patterns [3]. The first is an extension of $\mathrm{He}$ and Wang's texture spectrum to $5 \times 5$ windows, along with new structural features that capture local patterns such as horizontal and vertical stripes, alternating dark and bright spots, etc. The second is a new method that characterizes patterns as contrast variations in $5 \times 5$ windows.

\section{OVERVIEW}

As shown in fig 3.1, the user first submits query keyword. A pool of images is retrieved by text-based search. Then the user is asked to select a query image from the image pool.

System will extract features of query image. Images in the pool are re-ranked based on their visual similarities to the query image and the similarities are computed by comparing features with stored database image features.

Clusters of visually similar images are crated and cluster with most similar image is selected for visual expansion. This cluster images are added with retrieved resulting images and images are re-ranked. This will reflect the user's intention at finer level. capture user intention. 


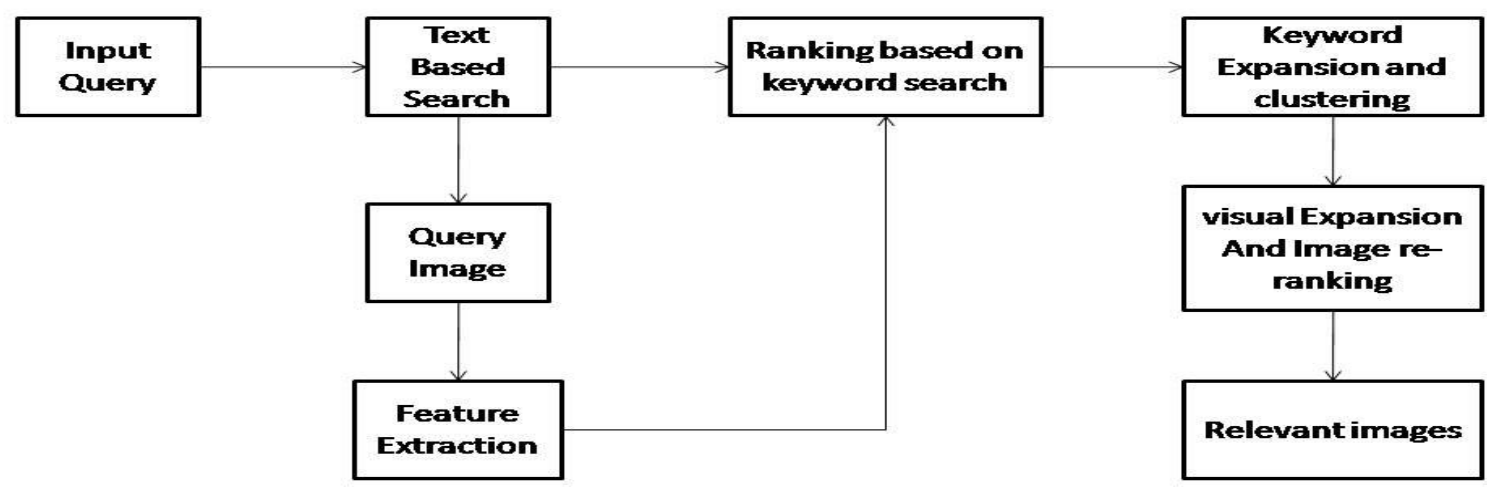

Fig 3.1 System Block Diagram

IV. SYSTEM IMPLEMENTATION

In this system, we implement four different module which distinct color.

together satisfies the intention of user. These modules are 3. Classify each pixel either as coherent or 1.Text based search, 2.Feature extraction, 3.Keyword incoherent. This is computed by

expansion and clustering, 4.Visual expansion and image re-ranking. This can be explained as follow,

\section{A. $\quad$ Text based search}

This module accepts keyword from user and retrieves the thousands of images, from which user clicks on one query image. Based on keyword enter by the user, it is searched into the image database for match with keyword, matching result of images is shown to user.

In this module, we used simple string matching algorithm to find out images with same label as text query.

\section{B. $\quad$ Feature Extraction}

To retrieve more relevant images from database, we used two feature extraction methods, that are- CCV and TEFC.

\section{1. $\quad C C V$}

Color is the most extensively used visual content for image retrieval. Its three-dimensional values make its discrimination potentiality superior to the single dimensional gray values of images.

$\mathrm{CCV}$ is a histogram-based method for comparing images that incorporates spatial information. It classifies each pixel in given color bucket as either coherent or incoherent, based on whether or not it is part of large similarly-colored region.

A color coherence vector $(\mathrm{CCV})$ stores the number of coherent versus incoherent pixels with each color. By separating coherent pixels from incoherent pixels, CCV provides finer distinction than color histogram.

\section{Algorithmic Steps for CCV:}

1. Blur the image (by replacing each pixel's value with the average value of the 8 adjacent pixels surrounding that pixel).
- Finding connected components for each discretized color.

- $\quad$ Determine tau's value (Tau is a user- specified value).

Any Connected Component has number of pixels more than or equal to tau then its pixels are considered coherent and the others are incoherent.

4. For each color compute two values ( $C$ and $N)$.

- $\quad C$ is the number of coherent pixels.

$N$ is the number of incoherent pixels.

\section{TEFC}

Texture is another important property of images. Various texture representations have been investigated in pattern recognition and computer vision. Basically, texture representation methods can be classified into two categories: structural and statistical. Structural methods, including morphological operator and adjacency graph, describe texture by identifying structural primitives and their placement rules. They tend to be most effective when applied to textures that are very regular.

Texture Element Feature Characterization (TEFC) is a structural method for texture analysis of CBIR. It uses local pattern as key and is expected to return more relevant images in CBIR when used as feature. Here, Local Patterns are characterised by using two methods:

Texture spectrum- It is a larger and visually more meaningful variation of $\mathrm{He}$ and Wang's approach which uses $3 * 3$ window size. It uses $5 * 5$ window. 


$$
\left(\begin{array}{ccccc}
\mathrm{a}_{0} & - & \mathrm{a}_{2} & - & \mathrm{a}_{4} \\
- & \mathrm{a}_{1} & \mathrm{a}_{3} & \mathrm{a}_{5} & - \\
\mathrm{a}_{14} & \mathrm{a}_{15} & \mathrm{a}_{\mathrm{c}} & \mathrm{a}_{7} & \mathrm{a}_{6} \\
- & \mathrm{a}_{13} & \mathrm{a}_{11} & \mathrm{a}_{9} & - \\
\mathrm{a}_{12} & - & \mathrm{a}_{10} & - & \mathrm{a}_{8}
\end{array}\right)
$$

Fig 4. I P1xel Index Values

The average intensity of the pixels along each direction is compared with that of central pixel. Hence, the reduction of $5 * 5$ window into a $3 * 3$ block is given mathematically by,

2 , if $\mathrm{a}_{2 \mathrm{i}}\left(\mathrm{w}+\mathrm{a}_{2 \mathrm{i}+1}{ }^{\mathrm{w}} 2 \mathrm{a}_{\mathrm{c}} \mathrm{w}^{\mathrm{w}}>0\right.$

$\mathrm{a}_{\mathrm{i}}^{\mathrm{U}}=\left\{1\right.$, if $\mathrm{a}_{2 \mathrm{i}}{ }^{\mathrm{W}}+\mathrm{a}_{2 \mathrm{i}+1}{ }^{\mathrm{W}}-2 \mathrm{a}_{\mathrm{c}}{ }^{\mathrm{W}}=0,0 \leq \mathrm{i}<7$

0 , if $\mathrm{a}_{2 \mathrm{i}}\left(\mathrm{W}^{\mathrm{w}}+\mathrm{a}_{2 \mathrm{i}+1}{ }^{\mathrm{W}}-2 \mathrm{a}_{\mathrm{c}}{ }^{\mathrm{W}}<0\right.$

The reduced block $U$ is identical to the $3 \times 3$ window used by He and Wang and we apply the same method proposed by them to generate a unique texture unit number.

$$
\tau(\mathrm{U})=\sum_{\mathrm{i}=0}^{7} \mathrm{a}_{\mathrm{i}}^{\mathrm{U}} 3^{\mathrm{i}}
$$

For any $M \times N$ pixel image, there will be $(M-2) \times(N-2)$ intensity patterns of size $5 \times 5$, each described uniquely by $\tau(U)$ such that $0 \leq \tau(U) \leq 6560$. Texture Unit Spectrum (TUS) ( $\mathrm{t}, 0 \leq \mathrm{t} \leq 6560)$, is the histogram of $\tau(\mathrm{U})$ 's within an image.

Contrast variation- It uses a set of patterns on $5 * 5$ windows based on contrast variation rather than intensity variation. It considers four major directions: $0^{\circ}, 45^{\circ}, 90^{\circ}$ and $135^{\circ}$. Each widow within an image is characterised by 4 directional contrast categories Contrast is measured by the difference in the sums of intensities of pixels lying on either side of a line drawn in the specified direction through the centre of the $5 \times 5$ window. For example, contrast at $45^{\circ}$ is measured as shown in Fig $2.2 a_{i}$ are always to the left as we walk in the direction of the line.

$$
\mathrm{C}_{45}=\sum_{\mathrm{i}=1}^{10} \mathrm{a}_{\mathrm{i}}-\sum_{\mathrm{i}=1}^{10} \mathrm{~b}_{\mathrm{i}}
$$

Contrast is then reduced to three categories: bright, equal or dark depending on whether it is greater than, equal to or less than 0 . In practice, contrast is considered equal if the difference is less than a predefined tolerance factor $\varepsilon$ expressed as a percentage of $\Sigma \mathrm{a}_{\mathrm{i}}$. Thus, each window in the image is characterized by the four directional contrast categories.Contrast features for the images form 12 dimensional feature vector given by 4 major directions each with 3 values (bright, equal and dark.).

\begin{tabular}{|l|l|l|l|l|}
\hline $\mathrm{a}_{1}$ & $\mathrm{a}_{2}$ & $\mathrm{a}_{3}$ & $\mathrm{a}_{4}$ & - \\
\hline $\mathrm{a}_{5}$ & $\mathrm{a}_{6}$ & $\mathrm{a}_{7}$ & - & $\mathrm{b}_{1}$ \\
\hline
\end{tabular}

\begin{tabular}{|c|l|l|l|l|}
\hline $\mathrm{a}_{8}$ & $\mathrm{a}_{9}$ & - & $\mathrm{b}_{2}$ & $\mathrm{~b}_{3}$ \\
\hline $\mathrm{a}_{10}$ & - & $\mathrm{b}_{4}$ & $\mathrm{~b}_{5}$ & $\mathrm{~b}_{6}$ \\
\hline- & $\mathrm{b}_{7}$ & $\mathrm{~b}_{8}$ & $\mathrm{~b}_{9}$ & $\mathrm{~b}_{10}$ \\
\hline
\end{tabular}

Fig 4.2 Calculating Contrast at $45^{\circ}$

\section{Keyword expansion and clustering}

In the keyword expansion step, words are extracted from the textual descriptions (such as image file names and surrounding texts in the html pages) of the top $\mathrm{k}$ images most similar to the query image, and the "term frequencyinverse domain frequency (tf-idf)" method is used to rank these words.

To save computational cost, only the top $m$ words are reserved as candidates for further processing. For each candidate word wi, we find all the images containing wi, and group them into different clusters based on visual content. For clustering we use k-means algorithm.

\section{Visual expansion and image re-ranking}

The image pool is enlarged through combining the original image pool retrieved by the query keywords provided by the user and an additional image pool retrieved by the expanded keywords. The size of the image cluster selected as visual query expansion and its similarity to the query image indicate the confidence that the expansion captures the user's search intention.

\section{EXPERIMENTAL RESULT}

In our experiment, we manually labeled 1000 images of 10 different categories, each category having 100 similar images.

After calculating CCV and TEFC features for each image, features of query image and database images are compared. According to the feature comparison, distance between query image and database images is calculated and stored in ascending order. This result is shown below. Fig 5.1(a) shows the result after color feature comparison. Fig 5.1(b) shows result after the texture feature comparison.

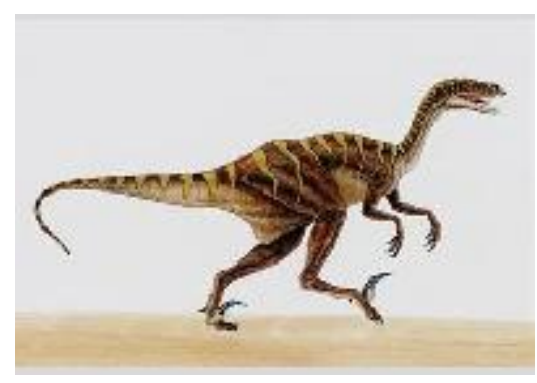

Fig 5.1 Input query image 


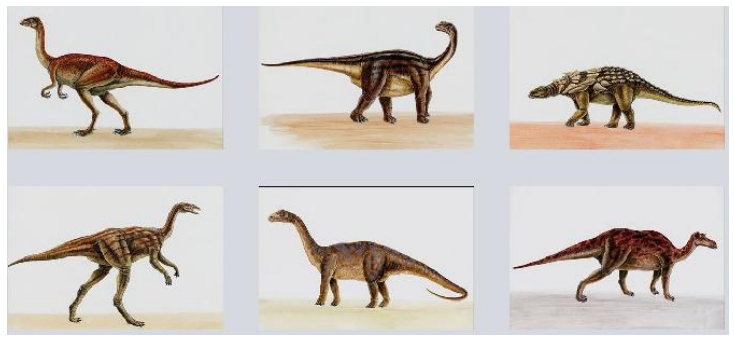

Fig 5.2(a) Result after CCV Comparison

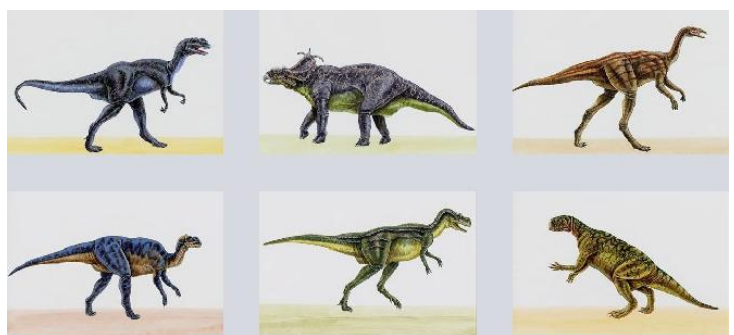

Fig 5.2(b) Result after TEFC Comparison

\section{CONCLUSION}

The image search system takes query input in the form of image and retrieves relevant images from huge database. In this system, we implement a novel Internet image search approach which only requires one-click user feedback. Without additional human feedback, textual and visual expansions are integrated to capture user intention. Expanded keywords are used to extend positive example images and also enlarge the image pool to include more relevant images.

For color features, we used color feature extraction method called Color Coherence Vector (CCV) which is an improvement over color histogram. For texture we used Texture Element Characterization (TEFC).

\section{REFERENCES}

[1] Xiaoou Tang, Ke Liu, Jingyu Cui,Fang Wen, " IntentSearch:Capturing User Intention for One-Click Internet Image Search", Pattern Analysis and Machine Intelligence, Vol.34 No.7 Year 2012.

[2] Greg Pass, Ramin Zabih, Justin Miller, “ Comparing Images Using Color Coherence Vectors ", Computer Science Department Cornell University Ithaca, NY 14853.

[3] K. Jalaja, Chakravarthy Bhagvati, B. L. Deekshatulu, Arun K. Pujari, "Texture Element Feature Characterizations for CBIR", Dept. of Computer and Information Sciences University of Hyderabad, Hyderabad 500046 (India).

[4] K. Arthi, J. Vijayaraghavan, "Content Based Image Retrieval Algorithm Using Colour Models", IJARCCE,Vol. 2, Issue 3, March 2013

[5] B. Dinakaran, J. Annapurna, Ch. Aswani Kumar, "Interactive Image Retrieval Using Text and Image Content", Bulgairian Academy of Sciences Cybernetics and Information Technologies, Volume 10, No 3, Year 2010 